Gdańsk 2019, Nr. 41

https://doi.org/10.26881/sgg.2019.41.15

Meike Meliss

Universität Santiago de Compostela \& Leibniz Institut für Deutsche Sprache Mannheim

https://orcid.org/000-0003-0380-8905

\title{
Und was kommt danach? Kontrastive Projekte und linguistische Pilgerreisen: Ein persönlicher Blick auf fast drei Dekaden Forschung von Ulrich Engel ${ }^{1}$
}

\begin{abstract}
Der Beitrag ist die Verschriftlichung der Laudatio, die die Autorin anlässlich des Festaktes zum 90. Geburtstag von Prof. Dr. Dr. h.c. mult. Ulrich Engel am Leibniz-Institut für Deutsche Sprache (=IDS) gehalten hat. Es handelt sich um eine persönliche Sicht auf fast drei Dekaden Forschungsarbeit, in denen zwischen Engel und der Germanistischen Abteilung der Universität Santiago de Compostela eine sehr enge Forschungskooperation bestand.
\end{abstract}

Schlüsselwörter: Ulrich Engel, Kontrastive Valenzlexikographie, Verbvalenz, Dependenzgrammatik, Kontrastive Grammatik

And what comes after that? Contrastive projects and linguistic pilgrimages: A personal look at almost three decades of research by Ulrich Engel. The article is a written version of the laudatory speech given by the author on the occasion of the $90^{\text {th }}$ birthday of Prof. Dr. Dr. h.c. mult. Ulrich Engel at the Leibniz Institute for German Language (=IDS). It is a personal view of almost three decades of research work in which Engel and the German Department of the University of Santiago de Compostela cooperated very closely.

Keywords: Ulrich Engel, contrastive valence lexicography, verb valence, dependency grammar, contrastive grammar

Sehr verehrte Damen, sehr verehrte Herren, liebe Kollegen und Kolleginnen, lieber Herr Direktor Prof. Dr. Lobin, liebe ehemalige IDS Direktoren: Herr Prof. Dr. Stickel und Herr Prof. Dr. Eichinger, liebe Familie Engel und lieber Herr Professor Dr. Engel:

1 Der vorliegende Text ist die leicht modifizierte Verschriftlichung der Laudatio für Prof. Dr. Dr. h.c. mult. Ulrich Engel, die die Autorin am 04.12.2018 am Leibniz-Institut für Deutsche Sprache in Mannheim anlässlich des Festaktes zu seinem 90. Geburtstag gehalten hat. Für den vorliegenden Text wurden die Merkmale des Gesprochenen größtenteils beibehalten. 
Ich wurde von Herrn Prof. Dr. Lobin und Herrn Prof. Dr. Kątny gebeten, zu diesem gegebenen feierlichen Anlass eine Festrede zu halten, die sich mit der Schaffensphase von Professor Engel nach seinem Ruhestand - also ab 1991 - befasst. $^{2}$

Für mich persönlich ist dies eine außerordentlich große Ehre, denn Herr Prof. Engel hat nicht nur meinen eigenen wissenschaftlichen Werdegang entscheidend geprägt, sondern auch den einer, oder besser gesagt, schon zweier - vielleicht gar dreier Generationen von Linguisten und Linguistinnen - nicht nur meiner Heimatuniversität (Santiago de Compostela) - sondern europaweit. ${ }^{3}$

Dies hat Jacqueline Kubczak schon vor zehn Jahren - genau an dieser Stelle zu dem 80. Geburtstag von Professor Engel - passend mit folgenden Worten festgestellt:

„Der räumliche Einflussbereich Ulrich Engels reicht von Finnland im hohen Norden, wo die Germanistik deutlich von der Valenz- und Dependenzgrammatik geprägt wurde (Nikula), bis nach Neapel im äußersten Süden Italiens, wo versucht wird, mit Hilfe der Engel'schen Unterscheidungen Klarheit in die Grammatikografie des Italienischen zu bringen (Bianco).“ (KuBCZAK 2009: 21)

Es ist mir auch eine große Freude, mit Ihnen hier heute meinen ganz persönlichen Blick auf dieses mehr als ein Viertel Jahrhundert Forschungsarbeit von Herrn Prof. Engel zu teilen. Meine Freude hat mindestens zwei Gründe: Erstens fällt diese Zeit zusammen mit der Entwicklung meines eigenen akademischen Werdegangs in Lehre und Forschung, der sicher ohne die Rezeption und den wissenschaftlichen Einfluss von Engel einen ganz anderen Weg eingenommen hätte und 1991 an der Universität Santiago de Compostela zeitlich verortet werden kann. Zweitens hatte ich das Privileg, genau in dieser Zeit Herrn Engel auch persönlich kennenzulernen und v.a. in den 90iger und Nullerjahren sehr intensiv mit ihm zusammen arbeiten zu dürfen.

Im Jahre 1991, als Herr Engel mit 63 Jahren von seinen Tätigkeiten am IDS in den Ruhestand verabschiedet wurde, handelte es sich sicher eher um einen symbolischen Akt. Denn Herr Engel legte sich ab 1991 keineswegs zur Ruhe - nein, nun legte er erst richtig los und führte Projekte, die er in seiner Zeit am IDS begonnen bzw. konzipiert hatte, tatkräftig und mit sehr viel Energie und Einsatzbereitschaft durch. Hier möchte ich Arbeiten in drei Bereichen hervorheben, die zwar schon alle während seiner berufstätigen Zeit am IDS eingeleitet wurden, in denen er aber besonders aktiv und engagiert in der Zeit nach seinem Ruhestand wurde: (i) Weiterentwicklung der Beschreibung der Syntax und Grammatik des Deutschen, (ii) Erstellung von kontrastiven Grammatiken sowie (iii) kontrastiven Valenzwörterbüchern/Lexika.

Die 1977 publizierte Syntax der deutschen Gegenwartssprache und die 1988 erstmals erschienene Deutsche Grammatik erfahren nach dem ,Ruhestand' von Engel völlig überarbeitete Neuauflagen (Syntax: ${ }^{3} 1994 /{ }^{4} 2009$; Grammatik: 2004/22009) bzw. inhaltliche Erweiterungen in entsprechenden Kapiteln der IDS Grammatik (ZIFONUN et al. 1997).

2 Siehe dazu die Festschrift, die Herrn Prof. Dr. Engel anlässlich seines 90. Geburtstages überreicht wurde (KąTNY (Hg.) 2018), sowie den Bericht von Janusz TABOREK (2019).

3 Dieser Beitrag ist im Rahmen des Forschungsprojekts COMBIDIGILEX entstanden (MINECO FEDER: FFI2015-64476-P: "Ministerio de Economía y Competitividad": Modalität 1), welches an der Universidad de Santiago de Compostela (Spanien) verortet ist. Die Autorin dieses Beitrages ist Projektantragstellerin und Koleiterin des Projekts. 
2002 erschien außerdem eine Kurze Grammatik der deutschen Sprache. Da bekanntlich in der Kürze die Würze liegt, dient dieses Werk besonders gut als Grundlage für den außeruniversitären Bereich.

Im Anschluss an die Kontrastive Grammatik deutsch-serbokroatisch (1986) hat sich Engel seit 1991 zusammen mit den Kollegen und Kolleginnen im Ausland verstärkt um die Erstellung weiterer kontrastiver Grammatiken gekümmert. Die Kontrastive Grammatik Deutsch-Rumänisch war zwar schon auf den Weg gebracht und konnte 1993 erscheinen (ENGEL et al. 1993), und auch für die Deutsch-Polnische kontrastive Grammatik waren zu dem Zeitpunkt schon die Weichen gestellt, doch gab es immerhin noch viele Jahre harte Arbeit, bis die ersten zwei Bände 1999 (ENGEL et al. 1999) erscheinen konnten.

Obwohl Engel zu seinem 60. Geburtstag in Bezug auf seine Zukunftspläne äußerte: „So wie ich das sehe, wird die deutsch-polnische Grammatik das letzte kontrastive Projekt sein, an dem ich mich versuche [...]" (Mrazović 1988: 27), erscheinen danach u.a. noch fünf Bände der Deutsch-Serbischen kontrastiven Grammatik zwischen 2012 und 2018. ${ }^{4}$

Neben den kontrastiven Grammatiken konzipierte und verfasste Engel nach seinem Ruhestand zusammen mit den Kollegen und Kolleginnen der Partnerländer auch mehrere kontrastive Verbvalenzwörterbücher. Zu seinem 70. Geburtstag äußerte er Folgendes:

„Nach dem neu entwickelten Verfahren entstehen zur Zeit fünf kontrastive Valenzlexika (deutsch-albanisch, deutsch-arabisch, deutsch-bulgarisch, deutsch-bosnisch/kroatisch/serbisch, deutsch-spanisch).“ (KĄTNY 1999: XI)

Nicht alle dieser Pläne konnten erfolgreich durgeführt und abgeschlossen werden, aber doch immerhin die meisten: So publiziert er u.a. 2009 das Wörterbuch zur Valenz Deutsch-Bosnisch/ Kroatisch/Serbisch (Djordjević / Engel 2009), 2010 erscheint in Santiago de Compostela der Prototyp eines kontrastiven spanisch-deutschen online-Wörterbuches zur Verbvalenz: Diccionario contrastivo de valencias verbales: español-alemán (DCVVEA) und 2013 das Wörterbuch zur Verbvalenz Serbisch-Deutsch (DJORDJEVIĆ / ENGEL 2013). Engel hat somit eine Reihe von kontrastiven lexikografischen Arbeiten zur Verbvalenz, die mit dem Valenzlexikon Deutsch-Rumänisch (ENGEL / SAVIN 1983) begonnen haben, bis heute unermüdlich fortgesetzt.

Zusätzlich zu den genannten grammatischen und lexikografischen Nachschlagewerken hat Engel seit seinem Ruhestand über 50 Artikel in Zeitschriften und Sammelbänden publiziert und dabei Themen wie u.a. Wort- bzw. Satzstellung, valenz- und dependenztheoretische Aspekte, Tempusfragen, Wortartenklassifizierung, Fragen in Verbindung mit einer kommunikativen Grammatik und anwendungsorientierte DaF-Bereiche untersucht und behandelt. Außerdem war Prof. Engel auch nach seinem symbolischen Ruhestand noch bis 2000 Honorarprofessor an der Universität Bonn und hat dort zahlreiche Qualifikationsarbeiten betreut.

Für diese hervorragenden wissenschaftlichen Leistungen ist Prof. Ulrich Engel mehrfach geehrt und gewürdigt worden: u.a. mit vier Ehrendoktortiteln ausländischer Universitäten (2000: Wrocław [Breslau]; 2002: Santiago de Compostela; 2008: Bukarest und

Vgl. Engel / Alanović (2012-2014), Urović et al. (2017), Engel et al. (2018). 
Hermannstadt) und auch durch mehrere Festschriften. ${ }^{5}$ Dazu kommen zwei Sammelbände, die im Rahmen von festlichen Veranstaltungen mit und um Engel in Santiago de Compostela und in Bukarest entstanden sind (Engel / Meliss (Hg.) 2004; STĂNESCU (Hg.) 2008).

Gleich zu Beginn dieser überaus aktiven Schaffensphase hat sich meine persönliche Begegnung mit Herrn Prof. Engel ergeben. Dies verdanke ich folgender Tatsache: Zur Feier des ersten Abschlussjahrgangs der Absolventen unseres damals neuen Studienganges ,Germanistische Philologie an der Universität Santiago de Compostela organisierte meine gerade erst ins Leben gerufene Abteilung für ,Germanistische Philologie eine Tagung zum Thema „Norm und Transgression“. Es sollten namhafte Kollegen und Kolleginnen aus Deutschland dazu eingeladen werden, denn wir wollten uns als recht ehrgeizige junge Auslandsgermanistik doch auch gerne im sogenannten ,Inland' bekannt machen und auf diese Weise den Weg für zukünftige wissenschaftliche Austauschmöglichkeiten und Kooperationen bahnen. Ich hatte die Aufgabe, einen Grammatikologen zu diesem Ereignis einzuladen. Sofort hatte ich die Idee, den Autor der „roten Grammatik“ nach Santiago zu holen. Ich hatte an der Universität Complutense in Madrid während meines Studiums in den 80iger Jahren schon bei Professor Eustaquio Barjau die Syntax von Engel kennengelernt und hatte mich in meinen Lehrveranstaltungen an der USC seit 1992 entschieden, nicht wie sonst in Spanien traditionellerweise üblich, die blaue Helbig/Buscha-Grammatik, ${ }^{6}$ sondern die rote Engel-Grammatik zu nutzen - die bald nur noch im Studentenjargon die „Rote Bibel“ genannt wurde. Diese Grammatik schien mir nicht nur für die Vermittlung im DaF-Unterricht mit angehenden DaF-Lehrern und Lehrerinnen viel strukturierter und daher besser geeignet, sondern sie half auch mir selber, die Strukturen meiner eigenen Muttersprache besser zu durchschauen. Viel mehr wusste ich allerdings nicht über diesen Linguisten. In einer Zeit, in der Internet noch nicht existierte, war es nicht so leicht, Herrn Prof. Engel postalisch ausfindig zu machen. Nach längerem Recherchieren konnte ich eine Adresse an der Universität Bonn finden und schickte ihm - sicher etwas naiv und blauäugig aus meiner heutigen Sicht - eine offizielle Einladung, an unserer Tagung, die 1994 in Santiago stattfinden sollte, 7 teilzunehmen. Große Hoffnungen auf eine positive Antwort machte ich mir nicht. Die Chance, dass ein so renommierter Wissenschaftler auf eine Einladung einer jungen - damals noch nicht promovierten Dozentin einer unbedeutenden, unbekannten Germanistik-Abteilung in Spanien - am Ende der Welt - positiv reagieren würde, war doch minimal! Umso größer war die Überraschung, als nach relativ kurzer Zeit eine positive Antwort von Herrn Professor Engel - natürlich per Post - eintraf. Er hatte die Einladung angenommen und mit diesem ersten Besuch an der Universität Santiago de Compostela 1994 begann eine sehr enge Zusammenarbeit mit ihm, die in unterschiedlichen Konstellationen bis heute andauert.

Engel selber kommentierte - sehr bescheiden - dieses erste Zusammentreffen in seiner Rede zur Verleihung der Ehrendoktorwürde in Santiago de Compostela 2002 wie folgt:

\footnotetext{
5 Mrazović (Hg.) 1988, Kątny (Hg.) 1999, Jurasz / KąTny / Tomiczek (Hg.) 2006, Eichinger / KubCZAK / Berens (Hg.) 2011, KąTNy (Hg.) 2018.

6 Gemeint ist die Deutsche Grammatik von den Autoren Helbig und Buscha, die seit 1972 in zahlreichen Auflagen erschienen ist und nach wie vor gerne im universitären DaF-Unterricht verwendet wird $\left({ }^{1} 1972\right)$.

7 Siehe dazu Millet (Hg.) 1994.
} 
„In dieser Stadt bin ich jetzt zum vierten Mal, und dass ich überhaupt hierher kam, verdanke ich eigentlich dem Leichtsinn meiner hiesigen Kollegen, die mich, ohne mich persönlich zu kennen, im Jahr 1994 zu einer Konferenz über Norm und Transgression in der deutschen Sprache und der deutschen Literatur eingeladen haben - und offenbar mit mir zufrieden waren. Mich jedenfalls hat die Stadt -, ja, und die Menschen in dieser Stadt und in diesem Land seither nicht mehr losgelassen. Heute kenne ich die Stadt recht gut, einigermaßen auch das Land Galicien." (ENGEL 2002: 16)

Man könnte sagen, dass sich die Kooperation mit Engel aus meiner compostelanen Perspektive heraus in folgenden drei Phasen manifestiert hat:

(i) Von 1995 bis 2002 hat sich die Zusammenarbeit mit ihm hauptsächlich in der Ausbildung und Förderung unseres wissenschaftlichen Nachwuchses geäußert. Engel wurde in das Doktorandenprogramm der Philologischen Fakultät der USC aufgenommen und kam mehrere Jahre hintereinander für Blockseminare und Gastvorträge an unsere Fakultät. Einige dieser Studierenden der ersten Jahrgänge unseres Germanistikstudiums sind heute Kollegen und Kolleginnen an unserer und anderen Fakultäten Spaniens und viele andere sind als DaF-Lehrende an Gymnasien und offiziellen Sprachenschulen - hauptsächlich in Galicien, aber auch landesweit und sogar in Deutschland - tätig. Die Betreuung von Dissertationsarbeiten, gemeinsame Publikationen (Engel / Meliss 2000), die Ausarbeitung eines Kapitels meiner eigenen Dissertation zu den theoretischen Grundlagen eines kontrastiven Ansatzes zur Beschreibung der syntaktischen und semantischen Valenz deutscher und spanischer Verben zum Ausdruck von Geräusch auf der Grundlage der engelschen Valenztheorie (Meliss 2005) und die Entwicklung erster Ideen zu der Erstellung eines Deutsch-Spanischen Verbvalenzwörterbuches gehören außerdem in diese Zeit und wirkten richtungsweisend für die zweite Phase der Zusammenarbeit. ${ }^{8}$

Dazu kam das Privileg, Engels wissenschaftliche Unterstützung und Betreuung bei den zahlreichen Forschungsaufenthalten am IDS zu genießen, die seit Mitte der 90-iger Jahre für einige von uns jungen Dozentinnen der USC und später auch für einige Nachwuchswissenschaftlerinnen möglich waren. Dank unterschiedlicher Förderungen aus Spanien und Deutschland und der freundlichen Aufnahme sowohl von einigen Wissenschaftlern und Wissenschaftlerinnen des Hauses als auch von den Kolleginnen in der Bibliothek wurden die vielen arbeitsintensiven Sommermonate in Mannheim zu einem unvergesslichen Forschungserlebnis.

Anzumerken ist aber auch, dass die direkte und indirekte Rezeption von Engels Werk in dieser Zeit nicht nur in Santiago de Compostela erfolgte, sondern parallel auch an anderen Orten der iberischen Halbinsel - so wurden u.a. für Prof. Engel Gastvorträge in Porto und Barcelona in Verbindung mit den Reisen nach Santiago organisiert. Mein Kollege von der Universität Tarragona, Andreu Castell, publizierte 1997 eine auf Spanisch verfasste Grammatik des Deutschen, die auf den valenz-dependenziellen Grundlagen der Grammatik von

8 Mit bescheidenem Stolz zitiere ich hier das Vorwort von Engel zu meiner lexikologischen Studie zu Geräuschverben: „Die Arbeit von Meike Meliss wird wegweisend sein auch für die Valenzlexikographie, die sich, nachdem sie die Kombinatorik der Verben im Griff zu haben glaubte (was sich als folgenschwerer Irrtum erwies), nun verstärkt und mit erheblichem Aufwand den inhärenten Bedeutungen zuwendet. Das spanisch-deutsche Valenzprojekt, das in Santiago de Compostela entsteht, wird davon profitieren." (Meliss 2005) 
Engel beruht, und in Portugal publizierte Mario Vilela 1999 eine Wort-, Phrasen- und Textgrammatik des Portugiesischen, die sich sehr an die Grammatik von Engel anlehnt. ${ }^{9}$

Nachdem Herrn Prof. Dr. Engel schon im Jahre 2000 die Ehrendoktorwürde von der Universität Wroclaw [Breslau] verliehen wurde, wurde ihm 2002 auch von der Universität Santiago de Compostela diese Ehre zuteil. Unser germanistisches Seminar und v.a. der Gründer und damalige Lehrstuhlinhaber, Prof. Dr. Carlos Buján, dankte Prof. Dr. Engel in seiner Laudatio neben seinen wissenschaftlichen Leistungen ganz besonders für sein unermüdliches Engagement in der Nachwuchsförderung, von der die germanistische Abteilung der USC nachhaltig und bis heute über mehrere Generationen hinweg profitieren sollte.

Da Prof. Dr. Engel die Ehrendoktorwürde in Santiago nur mit der Bedingung annehmen wollte, während seines Aufenthaltes neben den Feierlichkeiten v.a. das Arbeiten in den Vordergrund zu stellen, wurde parallel eine Tagung zu dem Thema „Valenz in Lexikon und Grammatik" organisiert. Die Beiträge sind in dem Sammelband Dependenz, Valenz und Wortstellung vereint (ENGEL / Meliss (Hg.) 2004). Diese Tagung bildete auch gleichzeitig den Auftakt für eine langjährige Forschungskooperation zwischen einigen Linguistinnen des germanistischen Seminars und Kolleginnen des Lehrstuhls für Spanische Sprache von Prof. Dr. Guillermo Rojo ${ }^{10}$ der Philologischen Fakultät der USC. Diese langjährige Kooperation stand ab 2002 unter der wissenschaftlichen Leitung von Prof. Dr. Engel und der organisatorischen Leitung der Kollegin der Spanischabteilung, Prof. Dr. Victoria Vázquez Rozas.

(ii) Diese Kooperation leitet die zweite Phase der Zusammenarbeit mit Professor Dr. Engel in Santiago ein. Das Ziel dieser sehr engen und intensiven Zusammenarbeit, die bis 2010 andauerte, war die Erstellung eines kontrastiven Verbvalenzwörterbuches für das Sprachenpaar Deutsch-Spanisch. Dieses Forschungsprojekt wurde von 2002 bis 2010 durch verschiedene Drittmittel der galicischen Landesregierung und des spanischen Bildungsministerium gefördert. ${ }^{11}$

Anders als in den bisherigen kontrastiven Valenzwörterbuchprojekten, die Engel geleitet hatte und für die er ein bestimmtes Beschreibungsmodell entwickelt hatte, welches auf verschiedene Sprachen im Kontrast mit dem Deutschen angewendet werden konnte, war es unser Ziel, nicht vom Deutschen als Ausgangssprache auszugehen, sondern von der Muttersprache des anvisierten Benutzers, also vom Spanischen. Es sollte hauptsächlich als Produktionswörterbuch für den hispanophonen DaF-Lernenden dienen. ${ }^{12}$ Außerdem wollten wir authentische Belege, möglichst korpusbasiert, als Illustration für den realen Sprachgebrauch anbieten. ${ }^{13}$ Diese - im Vergleich zu den anderen kontrastiven Valenzwörterbüchern, an denen Engel mitgewirkt hatte - neuartige Konzeption stellte uns allerdings auch vor schwierige Aufgaben und neue Herausforderungen. Neben der Anpassung der methodologischen und theoretischen

9 Die Rezeption der Valenz-Dependenzgrammatik auf der iberischen Halbinsel erfolgte allerdings schon früher u.a. auch über Romanisten wie Gerd Wotjak (WotjaK 1972; WotJaK / Regales (Hg.) 1988, WotJAK / Veiga (Hg. 1990)), der die Leipziger Schule vertrat (vgl. dazu auch BAÉz 1988, BusSE / VILELA 1986 und VIlela 1992).

10 Gemeint ist die ehemalige Abteilung „Lengua española“ der Philologischen Fakultät der USC.

11 Vgl. dazu weitere Information unter: https://gramatica.usc.es/proxectos/valencia/?lang=de\&id=desc ripcion.

12 Vgl. dazu u.a. LÜBKe 2004 und Meliss 2004

13 Vgl. dazu u.a. LüBke / Poulsen /VázQuez Rozas 2009 und LüBke / VázQuez Rozas 2011. 
Grundlagen an die Beschreibungsrichtung Spanisch-Deutsch, musste eine Methode entwickelt werden, die spanischen Verben semantisch und syntaktisch zu desambiguieren, um ihnen danach die entsprechenden Übersetzungsäquivalente des Deutschen zuzuordnen. ${ }^{14}$ In vielen gemeinsamen Sitzungen und Seminaren haben wir zusammen mit Ulrich Engel über unseren Entwürfen gebrütet, endlos und leidenschaftlich diskutiert und debattiert. Der kontrastive Blick auf die unterschiedlichen Fragestellungen hat uns dabei auf beiden Seiten die Augen für linguistische Phänomene geschärft. So hat z.B. Engel nach langer Diskussion mit uns seine ursprünglichen Nominal- und Adjektivalergänzungen in die sogenannten ,Prädikativergänzungen' in der Neuauflage seiner Grammatik 2004 umbenannt und den Typ der ,Modifikativergänzung' neu eingeführt. Lange Diskussionen über den anwendungsorientierten Nutzen der vorgeschlagenen Metasprache zur Beschreibung der semantischen Valenz führten jedoch - um nur ein Beispiel zu nennen - nicht zu den gewünschten Einigungen und bildeten den Keim für Entwicklungen, die dazu führten, dass im spanisch-deutschen Verbvalenzwörterbuch Entscheidungen methodologischer Art getroffen werden mussten, die nicht mit Engels Ausgangsidee zur Erstellung verschiedener kontrastiver Valenzwörterbücher mit Deutsch nach seinem Modell übereinstimmten.

Das Ergebnis unserer langjährigen Arbeit an dem kontrastiven spanisch-deutschen Verbvalenzwörterbuch, welches sich aus den besagten Gründen von den anderen kontrastiven Verbvalenzwörterbüchern von Engel unterscheidet, ist folgendes:

„Das kontrastive Verbvalenzwörterbuch Spanisch-Deutsch (Diccionario contrastivo de valencias verbales español-alemán DCVVEA) liefert eine Beschreibung der kombinatorischen Möglichkeiten von über hundert hochfrequenten Verben des Spanischen und ihrer deutschen Äquivalente und macht präzise Angaben zu ihren semantischen und syntagmatischen Eigenschaften. Die Abgrenzung von Bedeutungsvarianten für die polysemen spanischen Lemmata geht zum einen von vorliegenden lexikographische Beschreibungen aus, die an die Zielsetzungen des DCVVEA angepasst wurden, und stützt sich zum anderen auf das empirische Datenmaterial, das die syntaktische Datenbank Base de datos sintácticos del español actual (BDS) zur Verfügung stellt. [...] Das DCVVEA ist ein syntagmatisches Wörterbuch mit alphabetischer Struktur und Spanisch als Metasprache. Die Einträge beziehen sich auf die einzelnen Bedeutungsvarianten eines spanischen Verbs und werden mit authentischen Beispielen belegt. Den spanischen Verbvarianten werden deutsche Verben zugeordnet, die zu ihnen in einer vollständigen oder partiellen Äquivalenzrelation stehen. Die Ermittlung dieser Äquivalente stützt sich auf die Übersetzung der Korpusbeispiele. Die Valenzbeschreibung der spanischen und der deutschen Verbvarianten enthält funktionale, kategoriale und semantische Angaben zu den einzelnen Verbaktanten und explizite Hinweise auf kontrastiv relevante Unterschiede zwischen den Einheiten beider Sprachen." (https:// gramatica.usc.es/proxectos/valencia/?lang=de\&id=caracteristicas)

Mit Worten von Prof. Eichinger kann man sicher behaupten, dass sich Engel in dieser Zeit durch ein ganz bestimmtes „Alleinstellungsmerkmal“ ausgezeichnet hat, nämlich durch

„seine Fähigkeit, in den verschiedensten Sprachräumen Europas Partner gefunden oder zumindest angeregt zu haben, die auf Basis seines Entwurfs einer deutschen Grammatik vergleichend-kontrastive Grammatiken oder Wörterbücher verfasst und diese Projekte mit Eifer und Energie zu einem guten Ende gebracht haben. Die deutsch-rumänische, deutsch-serbokroatische und deutsch-polnische kontrastive Grammatik geben davon ebenso Zeugnis, wie Wörterbücher für das Italienische, das Ungarische, sowie

14 Vgl. dazu u.a. Domínguez VÁzQueZ et al. 2008a und 2008b. 
vergleichende Studien etwa zur Wortstellung und in diesem Zusammenhang entstandene Dissertationen. Es ist bemerkenswert, dass Ulrich Engel, wie das spanische Beispiel zeigt, von diesen Vorhaben noch immer nicht abgelassen hat." (EICHINGER 2011: $14 \mathrm{ff}$.)

(iii) Die Erstellung der lexikografischen Ressource Spanisch-Deutsch (DCVVEA) in Santiago de Compostela, die online konsultiert werden kann, ist gleichzeitig Ausgangspunkt für neue und unterschiedliche lexikografische Projekte, die ab 2010 durch entsprechende Drittmittelförderungen an dem germanistischen Seminar der USC eingeworben werden konnten. Hier beginnt die dritte Phase, in der der Einfluss von Engel deutlich ausstrahlt, aber verschiedene Richtungen einnimmt und Anlass für neue und eigene wissenschaftliche Wege ermöglicht. Dieser Einfluss hat im Laufe der Jahre einigen von uns Wissenschaftlerinnen, die wir in Santiago und - teilweise - in Mannheim mit Engel aufgewachsen, bzw. groß geworden sind, ermöglicht, verschiedene eigenständige Forschungsprojekte in und um valenztheoretische Fragestellungen mit lexikografischer Anwendungsperspektive durchzuführen. Die langjährige enge Zusammenarbeit mit Prof. Ulrich Engel hat uns im Laufe der Zeit die notwendige wissenschaftliche Reife verliehen, die auch eine Orientierung auf neue Gegenstandsbereiche, wie z.B. den Nominalbereich oder die Verbindung von Valenzgrammatik und Ansätzen der Konstruktionsgrammatik einerseits und der Framesemantik andererseits ermöglichte. ${ }^{15}$

Die germanistische Linguistik in Santiago de Compostela ist im Laufe der letzten mehr als 25 Jahre dank der äußerst großzügigen und engagierten wissenschaftlichen Betreuung von Prof. Dr. Engel herangereift. Eine schrittweise eingeleitete Emanzipation von Engel ermöglichte aber auch das Beschreiten neuer Wege. Heute ist die germanistische Linguistik in Santiago durch verschiedene Forschungsprojekte international sehr gut aufgestellt und gut vernetzt. Auf dieses internationale Potenzial hat Engel schon 2002 hingewiesen, als er in seiner Festrede zur Verleihung der Ehrendoktorwürde erklärte, was ihn mit Santiago verbindet:

„Und mir wurde, je länger ich nachdachte, auch klar, welch ungeheure Integrationskraft diese Stadt am Rande Europas seinerzeit hatte, weil sie ein Zentrum der europäischen Christenheit war, weil hier, auch hier und zumal hier, das Herz Europas schlug. Und wenn ich heute diese jungen Galicierinnen und Galicier sehe und höre, die Deutsch reden besser als unsereiner, weil sie in Frankfurt oder Köln oder München aufgewachsen sind, die zurückkamen, weil sie sich als Spanier oder Galicier fühlten, dann denke ich mir, dass gerade hier der europäische Gedanke lebt, dass diese Stadt mitsamt ihrer regionalen Prägung, die uns Binnenländer so fasziniert, diese an der Peripherie gelegene Stadt im Grunde viel europäischer ist als unsere großen Städte [...]. Und weil ich das so sehe, deshalb bin ich gerne hier, heute und ein andermal [...]." (ENGEL 2002: 17)

Dieses Potenzial, von dem Engel spricht, hat er durch seine langjährige Arbeit in Santiago direkt und indirekt gefördert und gestärkt.

Im Namen aller meiner Kollegen und Kolleginnen aus Santiago und aus anderen Teilen Spaniens, aber auch aus Süd-, Ost-, West- und Nordeuropa - und aus aller Welt - im Namen aller derer, die das Privileg hatten und weiterhin haben, mit Engel zu arbeiten und von ihm

15 Hier sind u.a. die Projekte DICONALE und COMBIDIGILEX zu nennen und verschiedene projektbezogene Publikationen, wie z.B. Cuartero Otal (2015), González Ribao (2015), Fernández MénDEZ (2015) und MeLiss (2015, 2016, 2018). 
zu lernen - im Namen von uns allen - danke ich Ihnen, Herr Prof. Engel, für die vielen Brücken, die Sie zu uns und mit uns gebaut haben - mit den vielen kleinen und großen Steinen, die Sie immer wieder in Ihrem Pilgergepäck auf dem Weg zu uns dabei hatten.

Ich wünsche Ihnen und Ihrer Frau alles denkbar Gute und weiterhin viel Leidenschaft bei all ihren kleinen und großen Projekten im privaten und wissenschaftlichen Bereich.

\section{Vielen Dank! Muchas Gracias! Moitas Grazas!}

\section{Bibliographie}

BaÉz SAN José, Valerio (1988): Fundamentos críticos de la gramática de dependencias. Madrid: Sintesis. Busse, Winfried / Vilela, Mario (1986): Gramática de Valências: apresentação e esboģo de aplicação à língua portuguesa. Coimbra: Livraria Almedina.

Cuartero Otal, Juan (2015): Bummel-Verben: Problem bei der Beschreibung ihres Kombinationspotenzials. In: ENGELBERG, Stefan et al. (Hg.), 301-316.

CASTELL, Andreu (1997): Gramática de la lengua alemana. Madrid: Editorial Idiomas.

DJORDJEvić, Miloje / Engel, Ulrich (2009): Wörterbuch zur Verbvalenz: Deutsch-Bosnisch, Kroatisch, Serbisch. München: iudicium.

DjoRdjević, Miloje / Engel, Ulrich (2013): Wörterbuch zur Verbvalenz Serbisch-Deutsch. München / Berlin: Sagner.

Domínguez Vázquez, María J. / Lübke, Barbara / Meliss, Meike / Paredes, Gemma / Poulsen, Pia / VázQuez, Victoria (2008a): Presentación del Diccionario contrastivo de valencias verbales: español-alemán. Primera parte: características generales del proyecto. In: Campos Souto, Mar et al. (Hg.) (2008): La lexicografía bilingüe y didáctica ayer y hoy. Coruña: Servizo de Publicacións. Universidade da Coruña (= Anexos revista de Lexicografía 8), 51-61.

Domínguez Vézquez, María J. / Lübke, Barbara / Meliss, Meike / Paredes, Gemma / Poulsen, Pia / VÁzquez, Victoria (2008b): Presentación del Diccionario contrastivo de valencias verbales: español-alemán. Segunda parte: problemas descriptivos particulares. In: CAmpos Souto, Mar et al. (Hg.) (2008): La lexicografia bilingüe y didáctica ayer y hoy. Coruña: Servizo de Publicacións. Universidade da Coruña (= Anexos revista de Lexicografía 8), 63-74.

Eichinger, Ludwig M. (2011): Ulrich Engel - Eine Einführung. In: Eichinger, Ludwig M. / Kubczak, Jacqueline / Berens, Franz Josef (Hg.): Dependenz, Valenz und mehr: Beiträge zum 80. Geburtstag von Ulrich Engel. Festschrift. Tübingen: Groos, 13-16.

Eichinger, Ludwig M. / Kubczak, Jacqueline / Berens, Franz Josef (Hg.) (2011): Dependenz, Valenz und mehr: Beiträge zum 80. Geburtstag von Ulrich Engel. Festschrift. Tübingen: Groos.

ENGEL, Ulrich ( $\left.{ }^{1} 1977,{ }^{3} 1993\right)$ : Syntax der deutschen Gegenwartssprache. Berlin: Erich Schmidt.

EngEL, Ulrich ( $\left.{ }^{1} 1988\right)$ : Deutsche Grammatik. Heidelberg: Julius Groos.

Engel, Ulrich (12004, 22009): Deutsche Grammatik. Neubearbeitung. München: iudicium.

ENGEL, Ulrich (2002): Discursos da investidura de D. Ulrich Engel como Doutor „Honoris Causa“. Santiago de Compostela: Universidad Santiago de Compostela.

ENGEL, Ulrich (2002): Kurze Grammatik der deutschen Sprache. München: iudicium. 
ENGEL, Ulrich (42009): Syntax der deutschen Gegenwartssprache. Völlig neu bearbeitete Auflage. Berlin: Erich Schmidt.

Engel, Ulrich / Alanović, Milivoj / Ninković, Sanja (2018): Deutsch-serbische kontrastive Grammatik. Teil 5. Hamburg: Kovač.

EngeL, Ulrich / Alanović, Milivoj (2012-2014): Deutsch-serbische kontrastive Grammatik. Teil 1-3. Hamburg: Kovač.

EngEL, Ulrich / IsBăşESCU, Mihai / STĂNeSCU, Speranța / Nicolae, Octavian (1993): Kontrastive Grammatik: deutsch-rumänisch. Erster Band. Heidelberg: Julius Groos.

EngeL, Ulrich / Meliss, Meike (2000): Sprachwissenschaftliche Einführungen. Ein Leitfaden für Studierende des Faches Deutsch als Fremdsprache und der Auslandsgermanistik zu Beginn des 21. Jahrhunderts. In: WierLACHER, Alois et al. (Hg.): Jahrbuch Deutsch als Fremdsprache 2000. München: iudicium, 119-241.

Engel, Ulrich / Meliss, Meike (Hg.) (2004): Dependenz, Valenz und Wortstellung. München: iudicium.

Engel, Ulrich / Mrazović, Pavica (Hg.) (1986): Kontrastive Grammatik Deutsch-Serbokroatisch: Deutsch-Serbokroatisch (2 Bd.). München: Sagner.

Engel, Ulrich / Rytel-Kuc, Danuta / Jurasz, Alina / Cirko, Lesław (1999): Deutsch-polnische kontrastive Grammatik. Band 1 und 2. Warszawa: Energeia.

ENGEL, Ulrich / SAVIN, Emilia (1983): Valenzlexikon Deutsch-Rumänisch (= Deutsch im Kontrast 3). Heidelberg: Julius Groos.

Engelberg, Stefan / Meliss, Meike / Proost, Kristel / Winkler, Edeltraud (Hg.) (2015): Argumentstruktur zwischen Valenz und Konstruktion (= Studien zur deutschen Sprache 68). Tübingen: Narr Francke Attempto Verlag.

FERNÁNDEZ MÉNDEZ, Manuel (2015): Argumentstrukturmuster und valenzgrammatisch orientierte Information im deutsch-spanischen Kontrast. Exemplarische Analyse der Lexikalisierungsmöglichkeiten des Konzepts entfüHren. In: Engelberg, Stefan et al. (Hg.), 353-364.

González Ribao, Vanessa (2015): Das lexikosemantische Paradigma der neuen medialen Kommunikationsverben im Sprachvergleich (Spanisch-Englisch-Deutsch) unter dem Blickwinkel der Argumentstruktur. In: ENGELbERG, Stefan et al. (Hg.), 341-352.

Helbig, Gerhard / Buscha, Joachim ('1972): Deutsche Grammatik: ein Handbuch für den Ausländerunterricht. Leipzig: Enzyklopädie Verlag.

Jurasz, Alina / KąTNY, Andrzej / TomiczeK, Eugeniusz (Hg.) (2006): Ulrich Engel: Gaudium in scientia linguarum: Ausgewäblte Schriften. Wroclaw / Dresden: Neisse.

KąTNY, Andrzej (Hg.) (1999): Das Deutsche von innen und von außen: Ulrich Engel zum 70. Geburtstag. Festschrift. Poznań: Wydawnictwo Naukowe UAM.

KąTny, Andrzej (Hg.) (2018): Valenz und Dependenz, Theorie und Praxis: Festschrift für Professor Ulrich Engel zum 90. Geburtstag (= Studia Germanica Gedanensia 39). Gdańsk: Wydawnictwo Uniwersytetu Gdańskiego.

KuвcZAK, Jacqueline (2009): Bericht: Ehrenkolloquium anlässlich des 80. Geburtstags von Prof. Dr. Dr. h.c. mult. Ulrich Engel. In: Sprachreport 1/2009, 21-24

LÜBKE, Barbara (2004): Was soll ein kontrastives Verbvalenzwörterbuch: Spanisch-Deutsch leisten? Teil 1. In: Enget, Ulrich / Meliss, Meike (Hg.): Dependenz, Valenz und Wortstellung. München: iudicium, 221-237. 
LÜBKe, Barbara / Poulsen, Pia / VÁzquez Rozas, Victoria (2009): La explotación de un corpus analizado sintácticamente en el Diccionario contrastivo de valencias verbales español-alemán [Datos para un análisis contrastivo de valencias verbales: diccionarios y corpus]. In: CANTOS Gómez, Pascual / SÁnchez Pérez, Aquilino (Hg.): A Survey on Corpus-based Research / Panorama de investigaciones basadas en corpus. Murcia, AELINCO (Asociación Española de Lingüística de Corpus), 51-64.

LÜBKe, Barbara / VÁzQuez Rozas, Vázquez (2011): Construcciones de entrar y salir y sus equivalentes alemanes. In: Sinner, Carsten / Hernández Socas, Elia / Bahr, Christian (Hg.): Tiempo, espacio y relaciones espacio-temporales. Nuevas aportaciones de los estudios contrastivos. Frankfurt/M.: Peter Lang, 115-129.

MeLISs, Meike (2004): Was soll ein kontrastives Verbvalenzwörterbuch: Spanisch-Deutsch leisten? Teil 2. In: Engel, Ulrich / MeLiss, Meike (Hg.): Dependenz, Valenz und Wortstellung. München: iudicium, 238-257.

MELISS, Meike (2005): Recursos lingüisticos alemanes relativos a GERÄUSCH y sus posibles correspondencias en español: Un estudio lexicológico modular-integrativo (= Studien zur romanischen Sprachwissenschaft und interkulturellen Kommunikation 16). Frankfurt/M.: Peter Lang.

Meliss, Meike (2015): Argumentstrukturen, Valenz und Konstruktionen. Eine korpusbasierte Studie deutscher und spanischer "Geruchsverben“ im Kontrast. In: ENGELBERG, Stefan et al. (Hg.), 317-339.

MeLIss, Meike (2016): La complementación en la lexicografía pedagógica del alemán y el español. Realidades, necesidades y posibilidades del presente y del futuro. In: CASTELL, Andreu (Hg.): Sintaxis y diccionarios. La complementación en alemán y en español. Bern, Berlin: Peter Lang, 9-55.

MeLIss, Meike (2018): Expansivergänzungen und Verbklassen: Eine inter- und intralinguale Annäherung. In: KĄTNY, Andrzej (Hg.), 35-53.

Millet, Victor (Hg.) (1994): Norm und Transgression in deutscher Sprache und Literatur: Kolloquium in Santiago de Compostela, 4.-7. Oktober 1995. München: iudicium.

Mrazović, Pavica (Hg.) (1988): Valenzen im Kontrast: Ulrich Engel zum 60. Geburtstag. Heidelberg: Groos.

StĂnescu, Speranta (Hg.) (2008): Sprachvergleich - Kulturvergleich: quo vadis, KGdr? München: iudicium.

Taborek, Janusz (2019): Festakt zum 90. Geburtstag von Prof. Dr. Dr. h.c. mult. Ulrich Engel. In: Sprachreport 1/2019, Mannheim: IDS, 50-51.

Urović, Annette et al. (2017): Deutsch-serbische kontrastive Grammatik. Teil 4. Hamburg: Kovač.

Vilela, Mário (1992): Gramática de Valências. Teoria e Aplicação. Coimbra: Livraria Almedina.

VIleLA, Mário (1999): Gramática da língua portuguesa: gramática da palavra, gramática da frase, gramática do texto / discurso. Coimbra: Livraria Almedina.

WotJAK, Gerd (1972): Zur kontrastiven semantischen Valenzanalyse (Arbeitsmaterial Nr. 15). Halle/S.: MLU Halle-Wittenberg, Sektion Sprach- u. Literaturwissenschaft.

Wotjak, Gerd / Regales, Antonio (Hg.) (1988): Studien zum Sprachvergleich deutsch-spanisch: Materialien der I. Internationalen Arbeitstagung zum Romanisch-Deutschen Sprachvergleich; Karl-Marx-Universität Leipzig, 05. u. 06.10.1987. Valladolid: Librera Sandoval. Spanischer Deutschlehrerverband (SDV). 
Wotjak, Gerd / Veiga, Alexandre (Coord.) (1990): La descripción del verbo español. Santiago de Compostela: Servicio de Publicaciónes da Universidade de Santiago de Compostela.

Zifonun, Gisela et al. (1997): Grammatik der deutschen Sprache. Berlin / New York: de Gruyter.

\section{Ressourcen}

DICONALE: http://www.usc.es/gl/proxectos/diconale/aleman/

COMBIDIGILEX: http://combidigilex.wixsite.com/deutsch

DCVVEA: Diccionario contrastivo de valencias verbales: español-alemán https://gramatica.usc.es/ proxectos/valencia/diccionario/

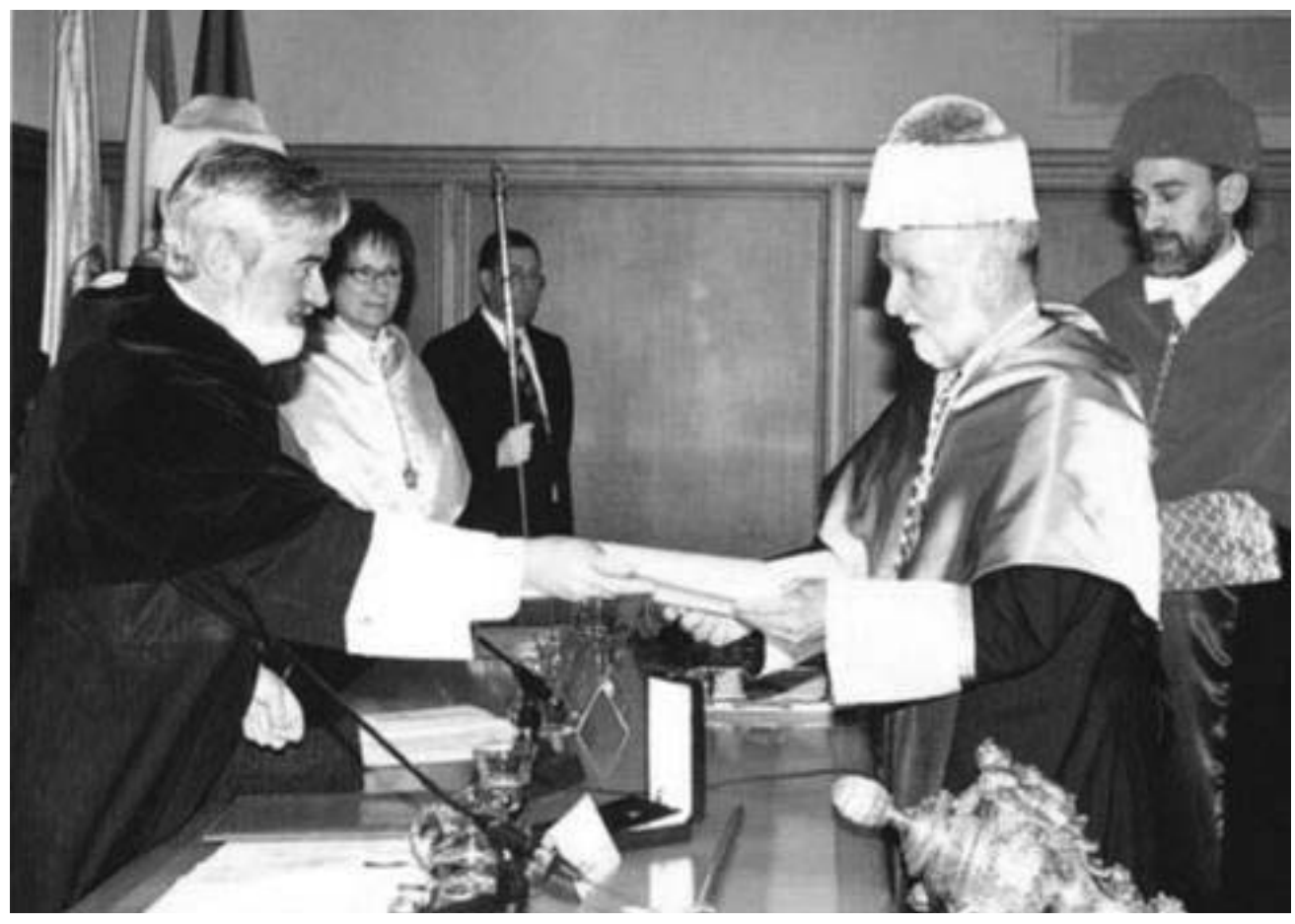

Verleihung der Ehrendoktorwürde der Universidad Santiago de Compostela an Prof. Dr. Ulrich Engel

Quelle: Engel 2002: Discurso [...] 